\title{
PNU-91325 increases fatty acid synthesis from glucose and mitochondrial long chain fatty acid degradation: a comparative tracer-based metabolomics study with rosiglitazone and pioglitazone in HepG2 cells
}

\author{
George G. Harrigan ${ }^{\mathrm{a}, *}$, Jerry Colca ${ }^{\mathrm{b}}$, Sándor Szalma ${ }^{\mathrm{c}}$, and László G. Boros ${ }^{\mathrm{d}}$ \\ ${ }^{a}$ Global High Throughput Screening (HTS), Pfizer Corporation, Chesterfield, MO 63017, USA \\ ${ }^{\mathrm{b}}$ Genomics and Biotechnology, Pfizer Corporation, Chesterfield, MO 63017, USA \\ ${ }^{\mathrm{c}}$ MeTa Informatics, San Diego, CA 92130, USA \\ ${ }^{\mathrm{d}}$ SIDMAP, LLC, Los Angeles, CA 90064, USA
}

Received 13 September 2005; accepted 4 January 2006

\begin{abstract}
The mitochondrial membrane protein termed "mitoNEET," is a putative secondary target for insulin-sensitizing thiazolidinedione (TZD) compounds but its role in regulating metabolic flux is not known. PNU-91325 is a thiazolidinedione derivative which exhibits high binding affinity to mitoNEET and lowers cholesterol, fatty acid and blood glucose levels in animal models. In this study we report the stable isotope-based dynamic metabolic profiles (SIDMAP) of rosiglitazone, pioglitazone and PNU-91325 in a dose-matching, dose-escalating study. One and $10 \mu \mathrm{M}$ concentrations 1 and $10 \mu \mathrm{M}$ drug concentrations were introduced into HepG2 cells in the presence of either $\left[1,2-{ }^{13} \mathrm{C}_{2}\right]$-D-glucose or $\left[\mathrm{U}-{ }^{13} \mathrm{C}_{18}\right]$ stearate, GC/MS used to determine positional tracer incorporation (mass isotopomer analysis) into multiple metabolites produced by the Krebs and pentose cycles, de novo fatty acid synthesis, long chain fatty acid oxidation, chain shortening and elongation. Rosiglitazone and pioglitazone $(10 \mu \mathrm{M})$ increased pentose synthesis from $\left[\mathrm{U}-{ }^{13} \mathrm{C}_{18}\right.$ ] stearate by $127 \%$ and $185 \%$, respectively, while PNU-91325 rather increased glutamate synthesis in the Krebs cycle by $113 \%$ as compared to control vehicle treated cells. PNU-91325 also increased stearate chain shortening into palmitate by $59 \%$. Glucose tracer-derived de novo palmitate and stearate synthesis were increased by 1 and $10 \mu \mathrm{M}$ rosiglitazone by $41 \%$ and $83 \%$, respectively, and by $63 \%$ and $75 \%$ by PNU-91325. Stearate uptake was also increased by $10 \mu \mathrm{M}$ PNU-91325 by $15.8 \%$. We conclude that the entry of acetyl Co-A derived from long-chain fatty acid $\beta$-oxidation into the mitochondria is facilitated by the mitoNEET ligand PNU-91325, which increases glucose-derived long chain fatty acid synthesis and breakdown via $\beta$-oxidation and anaplerosis in the mitochondria.
\end{abstract}

KEY WORDS: PNU-91325; mitoNEET; stable isotope-based dynamic metabolic profiling (SIDMAP); fatty acid oxidation; glucose metabolism; diabetes.

\section{Introduction}

Thiazolidinedione (TZD) compounds are used for the treatment of type 2 diabetes and are believed to exert their beneficial effects by increasing glucose-derived fatty acid synthesis secondary to the increased degradation of long chain fatty acids through the activation of the nuclear receptor peroxisome proliferator-activated receptorgamma (PPAR $\gamma)$. Studies with a tritiated photo-affinity labeled pioglitazone (1)<smiles>CCc1ccc(CCOc2ccc(CC3SC(=O)NC3=O)cc2)nc1</smiles>

analog identified a novel saturable TZD binding site also in mitochondria (Colca et al., 2004). The binding correlated with a $<17-\mathrm{kDa}$ protein, which showed selectivity of competition by analogs. The protein is termed

\footnotetext{
* To whom correspondence should be addressed.

E-mail: george.g.harrigan@monsanto.com
}

"mitoNEET," and has been found in the mitochondrial fraction of rodent brain, liver, and skeletal muscle. The protein also exists in low levels in preadipocytes, but expression increases exponentially in differentiated adipocytes (Colca et al., 2004).

Several pioglitazone (1) derivatives have been tested as antidiabetic TZDs for possible clinical development (Tanis et al., 1996). PNU-91325 (2)<smiles>CCc1ccc(C(=O)COc2ccc(CC3SC(=O)NC3=O)cc2)nc1</smiles>

was found to be the most effective TZD and proved to have greater binding to mitoNEET. Thus modification of mitoNEET function may, at least in part, contribute to lowering circulating lipid levels and glucose in the $\mathrm{KKA}^{\mathrm{y}}$ mouse model of human type-2 diabetes. Evidence has recently been presented suggesting that the insulin sensitizing effects of thiazolidinediones may correlate with modification of mitochondrial function rather 
than activation of PPAR $\gamma$ (Colca, 2006). Changes in mitochondrial function may alter the usage of cellular metabolites (e.g., sugars and fatty acids) in a way that favors insulin sensitivity.

In this study we report the stable isotope-based dynamic metabolic profile (SIDMAP) of rosiglitazone (3),<smiles>CN(CCOc1ccc(CC2SC(=O)NC2=O)cc1)c1ccccn1</smiles>

pioglitazone (1) and PNU-91325 (2) in a dose-matching, dose-escalating in vitro study. In order to follow substrate flow in the glucose metabolic network and the fate of the long chain fatty acid derived acyl-CoA we used two tracers; $\left[1,2-{ }^{13} \mathrm{C}_{2}\right]$-D-glucose or $\left[\mathrm{U}-{ }^{13} \mathrm{C}_{18}\right]$ stearate pool in differentiated primary hepatocellular carcinoma (HepG2) cells and gas chromatography/mass spectrometry (GC/MS) to analyze RNA ribose synthesis, lactate production, TCA cycle glutamate synthesis, as well as myristate, palmitate, stearate and oleate synthesis and turnover using the comprehensive multiple pathway tracer substrate flow analysis platform of the SIDMAParray (Boros et al., 2004). It is herein demonstrated that PNU-91325 (2) potently increases de novo long chain fatty acid synthesis from glucose, while it also increases mitochondrial breakdown of long-chain derived acyl-CoA via TCA cycle anaplerosis and glutamate synthesis. On the contrary, rosiglitazone (3) and pioglitazone (1) are more effective in re-routing stearate derived acyl-CoA towards glyconeogenesis and the synthesis of five carbon metabolites such as ribose in the pentose cycle. We conclude that the mitoNEET protein is a secondary TZD target and has a role in increasing mitochondrial long chain fatty acid derived acyl-CoA disposal through TCA cycle anaplerosis.

\section{Materials and methods}

Two non-radiating stable isotope tracer carbon labeled substrates were used in this metabolic flux analysis study: (a) $\left[1,2-{ }^{13} \mathrm{C}_{2}\right]-\mathrm{D}$-glucose was purchased with $>99 \%$ purity and $99 \%$ isotope enrichment for each position from Cambridge Isotope Laboratories, Inc. (Andover, MA, USA); and (b) $\left[\mathrm{U}-{ }^{13} \mathrm{C}_{18}\right]$-stearic acid (Spectra Stable Isotopes, Spectra Gases Inc., Branchburg, NJ). Rosiglitazone (3), pioglitazone (1) and PNU-91325 (2) were provided by Pfizer, Inc., under a material transfer agreement.

\subsection{Cells and cell culture}

Human liver hepatocellular (epithelial) carcinoma HepG2 cells (ATCC CRL11997) were purchased from American Type Culture Collection (ATCC, Manassas,
VA, USA). HepG2 cells have an average doubling time of $34 \mathrm{~h}$ in DMEM with $10 \%$ fetal bovine serum and $2.5 \%$ horse serum (Gibco/BRL, Gaitersburg, MD) in the presence of antibiotics. The cells were incubated at $37{ }^{\circ} \mathrm{C}, 5 \% \mathrm{CO}_{2}$ and $95 \%$ humidity and passed by using trypsin $0.25 \%$ (Gibco/BRL) no more than three times after receipt from the ATCC and prior to use in this study. HepG2 cells have previously been used in both in vitro and in vivo experiments and responded to treatment with troglitazone and other compounds with characteristic metabolic profile changes showing altered macromolecule synthesis and fatty acid cycling (Lee et al., 1998a). Tracer-labeled parallel cultures of HepG2 cells were used in this study as controls to compare vehicle (DMSO) treated cell cultures to rosiglitazone (3), pioglitazone (1) and PNU-91325 (2) treatment in an escalating regime of drug treatment.

Seventy-five percent confluent cultures of HepG2 cells were incubated in either $\left[1,2-{ }^{13} \mathrm{C}_{2}\right]$-D-glucose or $\left[\mathrm{U}-{ }^{13} \mathrm{C}_{18}\right]$ stearate-containing media $(100 \mathrm{mg} / \mathrm{dl}$ total glucose concentration $=5 \mathrm{mM} ; 50 \%$ isotope enrichment; or $0.5 \mathrm{mM}$ uniformly labeled stearate). Cells were plated at a density of $10^{6}$ per T75 culture flask and rosiglitazone (3), pioglitazone (1) and PNU-91325 (2) were added in two concentrations, 1 or $10 \mu \mathrm{M}$ dissolved in $10 \%$ DMSO - 90\% culture media. Control cultures were treated with vehicle (10\% DMSO) only. The doses of rosiglitazone (3), pioglitazone (1) and PNU-91325 (2) for the present study were selected based on in vitro experiments demonstrating that these drugs effectively control glucose levels via PPAR $\gamma$ activation in various human cell culture systems in the 1 to a $10 \mu \mathrm{M}$ dose range (for review: Otto et al., 2002). Drug treatments were carried out for $72 \mathrm{~h}$ in the presence of each tracer. Glucose and U-stearate ${ }^{13} \mathrm{C}$ tracer levels in the medium were monitored using a Cobas Mira chemistry analyzer (Roche Diagnostics, Pleasanton, CA, USA). Fresh, tracer enriched culture medium and drug treatments were applied every $24 \mathrm{~h}$, three times during the experiment, in order to provide for the cells with steady tracerenriched medium and drug doses throughout the $72 \mathrm{~h}$ experimental period. After $72 \mathrm{~h}$ the cells were scraped, spun and frozen at $-80^{\circ} \mathrm{C}$ until further processing as described below.

\section{2. $R N A$ ribose stable isotope studies}

For mass isotopomer analysis RNA ribose was isolated by acid hydrolysis of cellular RNA after Trizol (Invitrogen Life Technologies, Carlsbad, CA, USA) extraction from the cell pellets. Ribose was derivatized to its aldonitrile acetate form using hydroxylamine in pyridine with acetic anhydride (Supelco, Bellefonte, PA, USA) before mass spectral analyses. We monitored the ion cluster around the $m / z 256$ (carbons $1-5$ of ribose) (chemical ionization, CI) and $m / z 217$ (carbons $3-5$ of ribose) and $m / z 242$ (carbons $1-4$ of ribose) (electron 
impact ionization, EI) to determine molar enrichment and the positional distribution of ${ }^{13} \mathrm{C}$ in ribose. By convention, the base mass of ${ }^{12} \mathrm{C}$ compounds (with their derivatization agents) is given as $m_{0}$ as measured by mass spectrometry as described elsewhere (Katz et al., 1993). Ribose molecules labeled with a single ${ }^{13} \mathrm{C}$ atom on the first carbon position $\left(m_{1}\right)$ recovered from RNA were used to gauge the ribose fraction produced by direct oxidation of glucose through the G6PD pathway. Ribose molecules labeled with ${ }^{13} \mathrm{C}$ on the first two carbon positions $\left(m_{2}\right)$ were used to measure the fraction produced by transketolase. Doubly labeled ribose molecules $\left(m_{2}\right.$ and $\left.m_{4}\right)$ on the fourth and fifth carbon positions were used to measure molar fraction produced by triose phosphate isomerase and transketolase (Boros et al., 2002). Because transketolase has the highest metabolic control coefficient in the non-oxidative branch of the pentose cycle (Sabate et al., 1995), we use the term transketolase throughout the paper. It should be noted, though, that transketolase and transaldolase, besides other enzymes, all are participants in non-oxidative pentose cycle metabolism in human cells.

\subsection{Lactate}

Lactate from the cell culture media $(0.2 \mathrm{ml})$ was extracted by ethylene chloride after acidification with $\mathrm{HCl}$. Lactate was derivatized to its propylamine-heptafluorobutyrate ester form and the $m / z 328$ (carbons $1-3$ of lactate) (chemical ionization, CI) was monitored for the detection of $m_{1}$ (recycled lactate through the PC) and $m_{2}$ (lactate produced by the Embden-MeyerhofParnas pathway) for the estimation of pentose cycle activity (Lee et al., 1998b). In this study we recorded the $m_{1} / m_{2}$ ratios in lactate produced and released by HepG2 cells in order to determine pentose cycle activity and pentose cycling versus anaerobic glycolysis in response to 1 and $10 \mu \mathrm{M}$ rosiglitazone (3), pioglitazone (1) and PNU-91325 (2) treatments.

\subsection{Glutamate}

${ }^{13} \mathrm{C}$ label distribution in glutamate (glutamic acid), which is synthesized from $\alpha$-keto-glutaric acid of the TCA cycle, from tracer labeled glucose or stearate is suitable for determining mitochondrial glucose and fatty acid disposal via anabolic substrate use within the cycle, also known as anaplerotic flux. For glutamate extraction tissue culture medium $(1 \mathrm{ml})$ was first treated with $1 \mathrm{ml}$ of $6 \%$ perchloric acid and the supernatant was passed through a $3 \mathrm{~cm}^{3}$ Dowex-50 $(\mathrm{H}+)$ column. Amino acids were eluted with $15 \mathrm{ml} 2 \mathrm{~N}$ ammonium hydroxide. In order to separate glutamate from glutamine, the amino acid mixture was passed through a $3 \mathrm{~cm}^{3}$ Dowex-1 (acetate) column, and then collected with $15 \mathrm{ml} 0.5 \mathrm{~N}$ acetic acid. The glutamate fraction from the culture medium was converted to its trifluoroacetyl butyl ester
(TAB) (Leimer et al., 1977). Under EI conditions, ionization of TAB-glutamate produces two fragments, $\mathrm{m} /$ z 198 and $m / z 152$, corresponding to $\mathrm{C} 2-\mathrm{C} 5$ and $\mathrm{C} 2-\mathrm{C} 4$ of glutamate. Glutamate labeled on the 4-5 carbon positions indicates pyruvate dehydrogenase activity while glutamate labeled on the $2-3$ carbon positions indicates pyruvate carboxylase activity for the entry of glucose carbons to the TCA cycle. TCA cycle anabolic glucose utilization is calculated based on the $m_{1} / m_{2}$ ratios of glutamate (Lee et al., 1996).

\subsection{Lipid extraction and analysis}

Lipid extractions were performed using methods described elsewhere (Lowenstein et al., 1975). In brief, the Trizol extract (bottom layer) of cell pellets after RNA extraction were saponified with $500 \mu \mathrm{l}$ of $30 \%$ $\mathrm{KOH}-$ ethanol $(1: 1 \mathrm{v} / \mathrm{v})$ at $70{ }^{\circ} \mathrm{C}$ overnight. The aqueous phase was acidified, and fatty acids were extracted in petroleum ether and dried under a stream of nitrogen. Fatty acids were methylated with $0.5 \mathrm{ml} 0.5 \mathrm{~N} \mathrm{HCl}$ in methanol for GC/MS analysis. Palmitate, stearate and oleate were monitored at $m / z 270, m / z 298$ and $m / z 264$, respectively, with the enrichment of ${ }^{13} \mathrm{C}$ labeled acetyl units either from tracer labeled glucose or stearate, which reflect synthesis, elongation and desaturation of the new lipid fraction as determined by mass isotopomer distribution analysis (MIDA) of different isotopomers (Lee et al., 1991). Mass isotopomer distribution was determined using the method of Lee et al., which corrects for the contribution of derivatizing agent and ${ }^{13} \mathrm{C}$ natural abundance to the mass isotopomer distribution of the compound of interest. The calculated mass isotopomer distribution is expressed as molar fractions $(\mathrm{m} 0, \mathrm{~m} 1, \mathrm{~m} 2, \mathrm{~m} 3$, etc.), which are the fractions of molecules containing $0,1,2,3, \ldots{ }^{13} \mathrm{C}$ substitutions, respectively. Data reduction and regression analyses were performed using the computer software Microsoft Excel $^{\circledR}$ version 5.0.

\subsubsection{Determination of precursor enrichment}

$\beta$-oxidation of $\left[\mathrm{U}-{ }^{13} \mathrm{C}_{18}\right]$ stearate generates $\left[1,2-{ }^{13} \mathrm{C}_{2}\right]$ acetyl-CoA, which is subsequently used as a precursor for de novo lipogenesis, chain elongation, energy production and anaplerosis via the tricarboxylic acid cycle. Thus, $\beta$-oxidation can be assessed through the determination of $\left[1,2-{ }^{13} \mathrm{C}_{2}\right]$ acetyl-CoA enrichment in the different synthetic products.

\subsubsection{Acetyl-CoA enrichment for de novo synthesis from glucose}

The enrichment of the cytosolic acetyl-CoA pool used in de novo fatty acid synthesis was determined from the mass isotopomer distribution in palmitate and stearate using the $\left[1,2-{ }^{13} \mathrm{C}_{2}\right]$-D-glucose as the tracer. De novo synthesis produces these long chain saturated fatty acids with 2,4 , or $6{ }^{13} \mathrm{C}$ atoms (m2, $\mathrm{m} 4$, and $\left.\mathrm{m} 6\right)$. The 
distribution of these mass isotopomers has been previously shown to be a binomial distribution (Hellerstein, 1991; Lee et al., 1992). Thus, the acetyl-CoA enrichment may be obtained from the consecutive mass isotopomer ratio $\mathrm{m} 4 / \mathrm{m} 2=(n-1) p / q=3.5 p / q$, where $n$ is the number of acetyl units in palmitate $=8, p$ is the enrichment of $\left[1,2-{ }^{13} \mathrm{C}_{2}\right.$ ]acetyl-CoA, $q$ is the unenriched acetyl-CoA, and $p+q=1$.

\subsubsection{Acetyl-CoA enrichment from mitochondrial oxidation}

$\left[1,2-{ }^{13} \mathrm{C}_{2}\right.$ ]acetyl-CoA produced either from $\left[1,2-{ }^{13} \mathrm{C}_{2}\right]-$ D-glucose or $\left[\mathrm{U}-{ }^{13} \mathrm{C}_{18}\right]$ stearate from $\beta$-oxidation combines with oxaloacetic acid and forms citric acid in the mitochondria. Citrate participates in the tricarboxylic acid cycle, eventually forming $\left[\alpha-4,5-{ }^{13} \mathrm{C}_{2}\right]$ ketoglutarate and glutamate (Haber and Lapidot, 2001). The enrichment of the $\mathrm{m} 2$ component of the C2-C5 fragment generally reflects tricarboxylic acid cycle activity. However, it is well known that contribution from unlabeled glutamate from the cells in culture with glutamine-containing medium can substantially dilute the glutamate enrichment (Darmaun et al., 1988). Therefore, the enrichment of the $\left[4,5-{ }^{13} \mathrm{C}_{2}\right]$ glutamate reflects the lower limit of mitochondrial acetyl-CoA cycling and anaplerosis.

\subsubsection{Acetyl-CoA enrichment for chain elongation}

Enzymes involved in chain elongation from $\left[\mathrm{U}-{ }^{13} \mathrm{C}_{18}\right]$ stearate to longer chain fatty acids are located in the endoplasmic reticulum, mitochondria, and peroxisomes (Vance and Vance, 2002). Chain elongation of uniformly labeled stearate with $\left[1,2-{ }^{13} \mathrm{C}_{2}\right]$ acetyl-CoA produces characteristic $\mathrm{m}+18$ clusters that can be used to determine the acetyl-CoA enrichment according to the rules of combination of two labeled precursors. The ratio of $m+2$ to $m+0$ acetyl-CoA for chain elongation is given by the ratio of $m+20$ to $m+18$ in arachidate using the $\left[\mathrm{U}-{ }^{13} \mathrm{C}_{18}\right]$ stearate tracer. The enrichment of $\left[1,2-{ }^{13} \mathrm{C}_{2}\right]$ acetyl-CoA for each step of chain elongation was determined using the method described elsewhere using HepG2 cells as the in vitro model of fatty acid metabolism in the presence of $\left[\mathrm{U}-{ }^{13} \mathrm{C}_{18}\right.$ ] stearate (Wong et al., 2004).

\subsection{Gas chromatography/mass spectrometry (GC/MS)}

Mass spectral data were obtained on the HP5973 mass selective detector connected to an HP6890 gas chromatograph. The settings were as follows: GC inlet $250{ }^{\circ} \mathrm{C}$, transfer line $280{ }^{\circ} \mathrm{C}$, MS source $230{ }^{\circ} \mathrm{C}$, MS Quad $150{ }^{\circ} \mathrm{C}$. An HP-5 capillary column (30 m length, $250 \mu \mathrm{m}$ diameter, $0.25 \mu \mathrm{m}$ film thickness) was used for glucose, ribose, deoxyribose, glutamate and lactate analyses. Alternatively, a Bpx70 column (25 m length, $220 \mu \mathrm{m}$ diameter, $0.25 \mu \mathrm{m}$ film thickness, SGE Incorporated, Austin, TX) was used for fatty acid analysis with specific temperature programming for myristate
(C:14), palmitate $(\mathrm{C}: 16)$, stearate $(\mathrm{C}: 18)$, oleate $(\mathrm{C}: 18-$ $1)$, as well as $C: 20, C: 22$ and $C: 24$ fatty acid analyses.

\subsection{Data analysis and statistical methods}

Each experiment was carried out using triplicate cell cultures for each condition within each experiment and experiments were repeated once. Mass spectroscopic analyses were carried out by three independent automatic injections of $1 \mu \mathrm{l}$ samples by the automatic sampler and accepted only if the standard sample deviation was less than $1 \%$ of the normalized peak intensity. Statistical analysis was performed using the Student's $t$-test for unpaired samples. Two-tailed significance at the $99 \%$ confidence interval $(\mu \pm 2.58 \sigma), p<0.01$ indicated significant differences in glucose carbon metabolism in control and rosiglitazone (3), pioglitazone (1) and PNU91325 (2) treated HepG2 cells. SIDMAParray ${ }^{\mathrm{TM}}$ was produced by MeTa Informatics (San Diego, CA).

\section{Results}

In this study the stable isotope-based metabolic profiles of rosiglitazone (3), pioglitazone (1) and PNU91325 (2) treated HepG2 cells are presented using two tracers, $\left[1,2-{ }^{13} \mathrm{C}_{2}\right]$-D-glucose and $\left[\mathrm{U}-{ }^{13} \mathrm{C}_{18}\right]$ stearic acid in separate experiments. The metabolic profiles reported in the SIDMAParrays present tracer carbon flow changes in all major metabolic pathways, which include glycolysis, the pentose and TCA cycles, de novo fatty acid synthesis, chain elongation, chain shortening and desaturation, in a color scheme similar to gene expression studies.

The SIDMAParray using the $\left[1,2-{ }^{13} \mathrm{C}_{2}\right]$-D-glucose tracer is shown in figure 1 . It can be seen from the array represented in figure 1 that rosiglitazone (3) and PNU91325 (2) induced a significant increase in de novo fatty acid synthesis from glucose, including myristate, palmitate and stearate (box 3), while substrate flux through the pentose cycle producing ribose and deoxyribose were less affected by either drug (boxes 1 \& 2). Rosiglitazone (3) and PNU-91325 (2) increased de novo stearate synthesis from glucose by $41 \%$ and $83 \%$ and by $63 \%$ and $75 \%$ using 1 and $10 \mu \mathrm{M}$ doses, respectively. It can also be seen from the array that all compounds increased glucose-derived acetyl-CoA ${ }^{13} \mathrm{C}$ enrichment of myristate and stearate. There were no significant changes in pentose cycle direct glucose oxidation and recycling into lactate or TCA cycle anaplerotic flux for the synthesis of glutamate from glucose (data not shown). It can be concluded from the array that 1 and $10 \mu \mathrm{M}$ rosiglitazone (3) and PNU-91325 (2) induced similar changes in glucose carbon utilization in HepG2 cells, which was consistent with increased de novo long chain fatty acid synthesis from glucose, with little or no effect on the rate of glucose oxidation $\left({ }^{13} \mathrm{CO}_{2}\right.$ release in the TCA and 


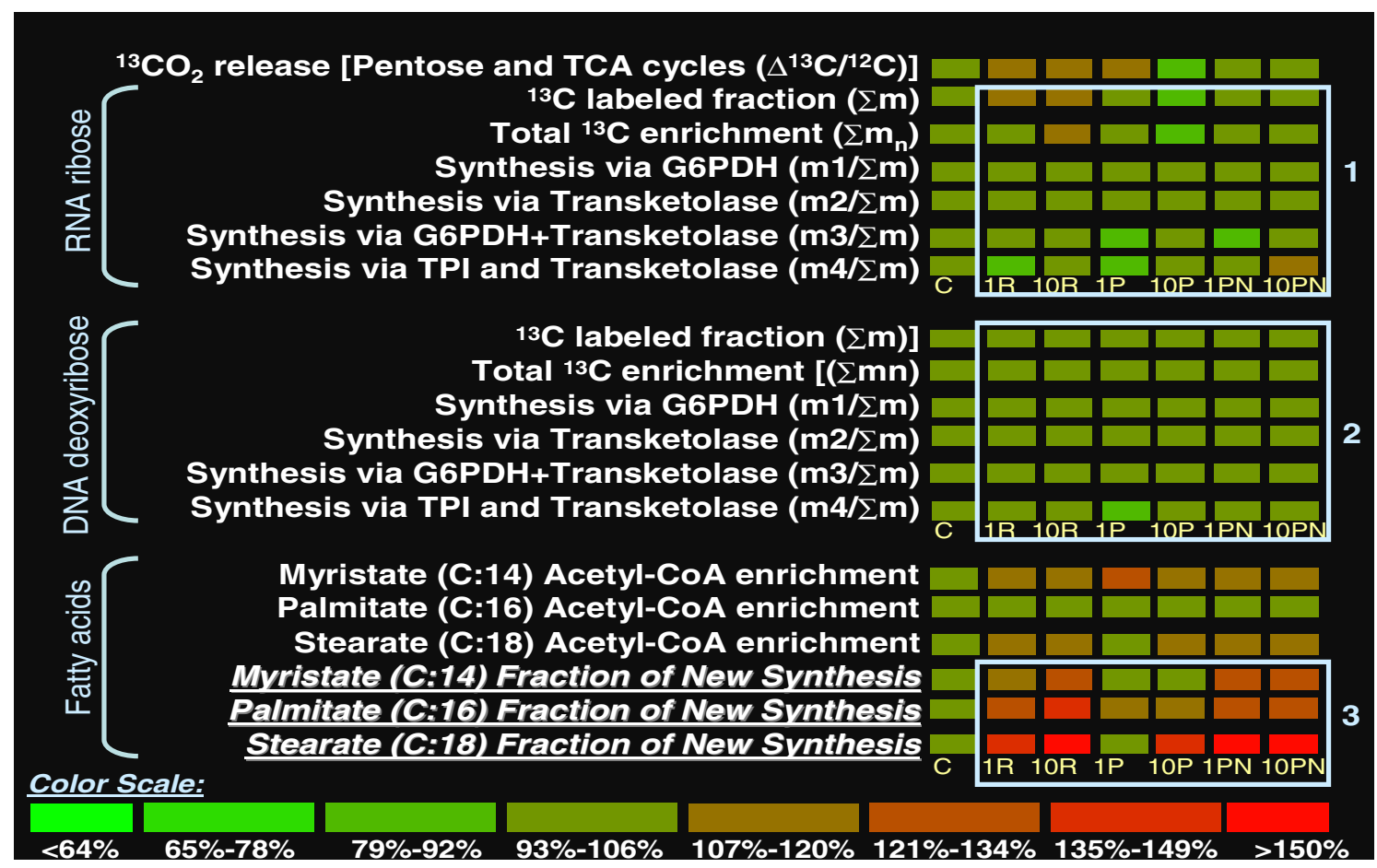

Figure 1. Stable isotope-based dynamic metabolic profile array (SIDMAParray) of rosiglitazone, pioglitazone and PNU-91325 in HepG2 cells using $\left[1,2-{ }^{13} \mathrm{C}_{2}\right]$-D-glucose as the tracer. Treatments are indicated as follows: $\mathrm{C}=\mathrm{Control} ; 1 \mathrm{R}=1 \mu \mathrm{M}$ rosiglitazone; $10 \mathrm{R}=10 \mu \mathrm{M}$ rosiglitazone; $1 \mathrm{P}=1 \mu \mathrm{M}$ pioglitazone; $10 \mathrm{P}=10 \mu \mathrm{M}$ pioglitazone; $1 \mathrm{PN}=1 \mu \mathrm{M}$ PNU-91325; and 10PN $=10 \mu \mathrm{M}$ PNU-91325. The color of each square corresponds to ${ }^{13} \mathrm{C}$ tracer glucose flux via various synthetic pathways of ribose, deoxyribose and fatty acids as percent of control-DMSO treated cultures. A color scheme indicating the intensity of changes in substrate flux is provided in the bottom of the figure. Significant increase in de novo fatty acid synthesis from glucose are underlined and italicized $(\mathrm{G} 6 \mathrm{PDH}=$ glucose 6-phosphate dehydrogenase; TPI $=$ Triose-phosphate isomerase).

pentose cycles), or de novo ribose and deoxyribose synthesis in the pentose cycle. Ten $\mu \mathrm{M}$ pioglitazone (1) was also effective in increasing de novo stearate synthesis from glucose by about $30 \%$ of control cells.

There were more characteristic and obvious differences in how rosiglitazone (3), pioglitazone (1) and PNU-91325 (2) affected uniformly labeled stearate synthesis as figure 1 .

The main difference in stearate utilization among the drugs is that 1 and $10 \mu \mathrm{M}$ rosiglitazone (3) and pioglitazone (1) induced a dose dependent increase in pentose cycle ribose ${ }^{13} \mathrm{C}$ enrichment by $101 \%, 127 \%$ and $250 \%, 285 \%$ (box 1), while 1 and $10 \mu \mathrm{M}$ PNU91325 rather increased mitochondrial TCA cycle glutamate synthesis from stearate by $25 \%$ and $113 \%$ (box 2), respectively. Glutamate ${ }^{13} \mathrm{C}$ enrichment from stearate was only increased using $10 \mu \mathrm{M}$ rosiglitazone (3) or pioglitazone (1) by $22 \%$ and $21 \%$, respectively. It appeared that the primary route by which stearate ${ }^{13} \mathrm{C}$ tracer carbons entered the TCA cycle is via citrate synthesis, as $10 \mu \mathrm{M}$ pioglitazone (2) and PNU91325 (1) induced a $12 \%$ and $34 \%$ increase in glutamate synthesis via citrate (box 3 ). De novo palmitate synthesis from stearate-derived $\left[{ }^{13} \mathrm{C}_{2}\right]$ acetyl-CoA was increased by $102 \%$, while stearate chain shortening into palmitate was also increased by $59 \%$ using
$10 \mu \mathrm{M}$ PNU-91325 (box 4). Stearate uptake and chain elongation into arachidonic acid was also increased by $10 \mu \mathrm{M}$ PNU-91325 by $16 \%$ and $8 \%$, respectively (box 6).

Interestingly, the ${ }^{13} \mathrm{C}$ labeled fraction of deoxyribose (DOR) of DNA from stearate was decreased by $20 \%$ and $37 \%$ and total ${ }^{13} \mathrm{C}$ enrichment of DOR was also strongly inhibited by $24 \%$ and $45 \%$ using 1 and $10 \mu \mathrm{M}$ PNU91325 (box 6). On the other hand, there was no significant inhibition of deoxyribose synthesis from stearate by rosiglitazone (3) or pioglitazone (1) in the $72 \mathrm{~h}$ study period. There was about $10 \%$ increase in lactate ${ }^{13} \mathrm{C}$ enrichment from stearate using $1 \mu \mathrm{M}$ rosiglitazone (3) only, indicating that stearate derived acetyl-CoA disposal via lactate is not a significant route imposed by either drug.

\section{Discussion}

Metabolic profiling represents a logical development in the application of genomics, transcriptomics and proteomics in drug discovery and development. This study highlighted the relevance and robustness of stable isotope tracer-based dynamic metabolic profiles when applied to drugs discovered through reverse 
pharmacology (Harrigan et al., 2005). This study makes used of continued development of new approaches to generate and interrogate metabolome data sets. Here we have evaluated three thiazolidinediones that exhibit overall similar insulin sensitizing pharmacology in vivo but vary substantially in their ability to activate the transcription factor $\operatorname{PPAR} \gamma$. This study determined glucose and stearate flow redistribution among major metabolic pathways involving glycolysis, the pentose and TCA cycles, de novo fatty acid synthesis and degradation in response these three thiazolodinediones in living HepG2 primary liver tumor cells. The compounds tested in this study include two drugs marketed for the treatment of diabetes and a third analog that has potent insulin sensitizing activity without activating PPAR $\gamma$ regulated gene transcription (Colca, 2006). Gene transcription analysis from earlier studies suggests that changes the expression of mitochondrial proteins might be a key factor in the insulin sensitizing pharmacology. Our findings using glucose as a metabolic tracer are that rosiglitazone (3), pioglitazone (1) and PNU-91325 (2) increase de novo synthesis of various fatty acids from glucose, and that PNU-91325 (2) surpasses the effect of both marketed drugs. Using stearate as a metabolic tracer it is obvious that rosiglitazone (3) and pioglitazone (1) primarily induce fatty acid breakdown by increasing glyconeogenesis and RNA pentose synthesis, while PNU-91325 (2) forces stearate-derived acetyl units into the mitochondria via citrate synthesis in a dose dependent manner.

The main implication of our SIDMAP study is that predominantly PPAR $\gamma$ and predominantly mitochondrially acting (mitoNEET ligand) compounds direct long chain fatty acid catabolism into different routes. PNU91325 (2) is very effective in promoting mitochondrial stearate $\beta$-oxidation, while concomitantly increasing stearic acid uptake, chain shortening into palmitate and chain elongation into arachidonate. On the other hand, rosiglitazone (3) and pioglitazone (1) primarily increase pentose sugar synthesis from stearate, which indicates peroxisomal fatty acid metabolism and the production of acyl-CoA, which may enter the mitochondria and produce glutamate, but only at the high $10.0 \mu \mathrm{M}$ dose in this dose matching study with PNU-91325. The potency of PNU-91325 (2) in increasing mitochondrial betaoxidation of stearate and its disposal via glutamate is about 10 times higher than that of rosiglitazone (3) and pioglitazone (1). In previous in vivo experiments high resolution 2-D gel electrophoresis of livers showed that rosiglitazone (3) primarily produced changes in the expression pattern of many proteins involved in peroxisomal fatty acid $\beta$-oxidation (Edvardsson et al., 1999). Although rosiglitazone (3) and PNU-91325 (2) tend to increase the expression of many mitochondrial proteins and produce changes that favor fatty acid oxidation, PNU-91325 (2) does not significantly increase PPAR $\gamma$ driven transcription either in vitro or in vivo (Colca,
2006). The most likely explanation for the effects of these compounds on gene expression and on the metabolic fluxes observed in these studies is that the overall effect of a given analog represents a blend of direct mitochondrial actions (e.g., binding to mitoNEET) and effects on other sites of binding (e.g., PPAR $\gamma$ ).

It has been demonstrated that both PPAR $\gamma$ ligands investigated in this study, pioglitazone (1) and rosiglitazone (3) effectively decreased liver cancer formation using a diethylnitrosamine-induced liver cancer in Wistar rats (Guo et al., 2004) and colon carcinogenesis induced by azoxymethane in mice (Osawa et al., 2003). In our substrate flow tracer study, while rosiglitazone (3) and pioglitazone (1) increased RNA pentose synthesis from stearate, there was a decrease in tracer accumulation into DNA deoxyribose during the $72 \mathrm{~h}$ treatment period using these TZDs. On the other hand, DNA deoxyribose synthesis was strongly suppressed by PNU91325 (2) treatment from stearate. The glucose tracer study indicated unaltered rate of RNA and DNA ribose synthesis, which confirms the chemo-preventive effect of TZD based on direct substrate flow measurements via all arms of the pentose cycle.

We acknowledge two potential limitations of this study. First, because we studied primary hepatoma cells, it is not known how the studied compounds affect normal liver cell function and other tissue cells in a complex host. It is important to note that HepG2 cells grow rapidly in culture and they have higher specialized requirements for energy and macromolecule synthesis than primary cells. Conditions to test primary liver cells are under development. Metabolic labeling studies with primary cells are complicated by the fact that freshly isolated hepatocytes survive for $24 \mathrm{~h}$ providing limited time to produce a comprehensive SIDMAParray using either glucose or fatty acid tracers. In any case, HepG2 cells have maintained biotransformation properties of drugs and they also posses and express metabolic enzymes in a comparable manner with primary cultures of hepatocytes (Wilkening et al., 2003), while providing a highly viable and stable cell line for continuous tracer based metabolomics studies.

Second, it is currently not known what primary acylCoA species are produced from the tracer stearate substrate in response to the PPAR $\gamma$ agonist rosiglitazone (3) and pioglitazone (1) treatments. Based on assessing heavy incorporation of tracer carbons of stearate into the five carbon metabolite pool of ribose with no measurable tracer accumulation in lactate or palmitate it is likely that short chain fatty acids (C:4-C:6) are heavily produced and shuttled into the cytosol of HepG2 cells from the peroxisomes. As the use of $\mathrm{C} 18-\mathrm{C} 20$ chain elongation and other mass isotopomer analysis is the first quantitative method to determine peroxisomal acetyl-CoA enrichment (and therefore peroxisomal $\beta$-oxidation) (Wong et al., 2004), we will be able to identify those acyl-CoA pools in subsequent SIDMAP 
studies. Such studies should make use of the tracer method for both peroxisomal oxidation and very long chain fatty acid synthesis in HepG2 cell cultures together with animal experiments.

It is a particularly significant finding that rosiglitazone (3) and pioglitazone (1) treatments strongly increase pentose formation from tracer stearate, thereby increasing NADPH production and peroxisomal function. In the mammalian liver many $\beta$ - and per-oxidation related events require NADPH as the reducing equivalent. For example, peroxisomal $\beta$-oxidation produces hydrogen peroxide. Although hydrogen peroxide is not itself a radical, it is considered a reactive oxygen species because it is easily converted into hydroxyl radical, which has to be scavenged by NADPH-dependent reductases thus preventing cell damage. NADPH-dependent peroxidases are also important in the microsomal function of liver cells, therefore, increased carbon flow by rosiglitazone (3) and pioglitazone (1) towards the pentose cycle are expected during increased peroxisomal and microsomal fatty acid degradation (figure 2). In this paper we show direct evidence via net tracer carbon flow measurements from stearate towards pentoses that such link exists and such PPAR $\gamma$ ligands concomitantly increase peroxisomal degradation and pentose cycle metabolism in the same cells. These differences in metabolic flux from those produced by PNU-91325 (2) suggest that there will be some differences in the pharmacology produced by compounds that lack inherent PPAR $\gamma$-like activity.

In summary, we evaluated glucose and fatty acid metabolism based on two separate metabolic tracer substrate experiments using cultured human primary liver carcinoma cells. Whatever the genetic and proteomic mechanisms are in the complex array of genes and proteins altered by TZDs, PNU-91325 (2) unquestionably generates a dramatically different substrate flow profile from the long chain fatty stearate than do rosiglitazone (3) and pioglitazone (1). PNU-91325 (2) directs $\beta$-oxidation of stearate and chain shortening by one acetyl unit resulting palmitate, then this acetyl unit participates in chain elongation and TCA cycle glutamate synthesis. Rosiglitazone (3) and pioglitazone (1) rather produce short chain fatty acids and their shortening is strongly linked with pentose synthesis (figure 3).

Based on our current findings, PNU-91325 (2) is a more potent inducer of mitochondrial fatty acid $\beta$-oxidation and TCA cycle anaplerosis than are the existing compounds to treat hyperlipidemic conditions in type 2 diabetes with desirable anti-proliferative effects and limited DNA deoxyribose synthesis in liver cells.

In order to reveal organ and tissue specific metabolic changes involving liver, muscle, adipose and other

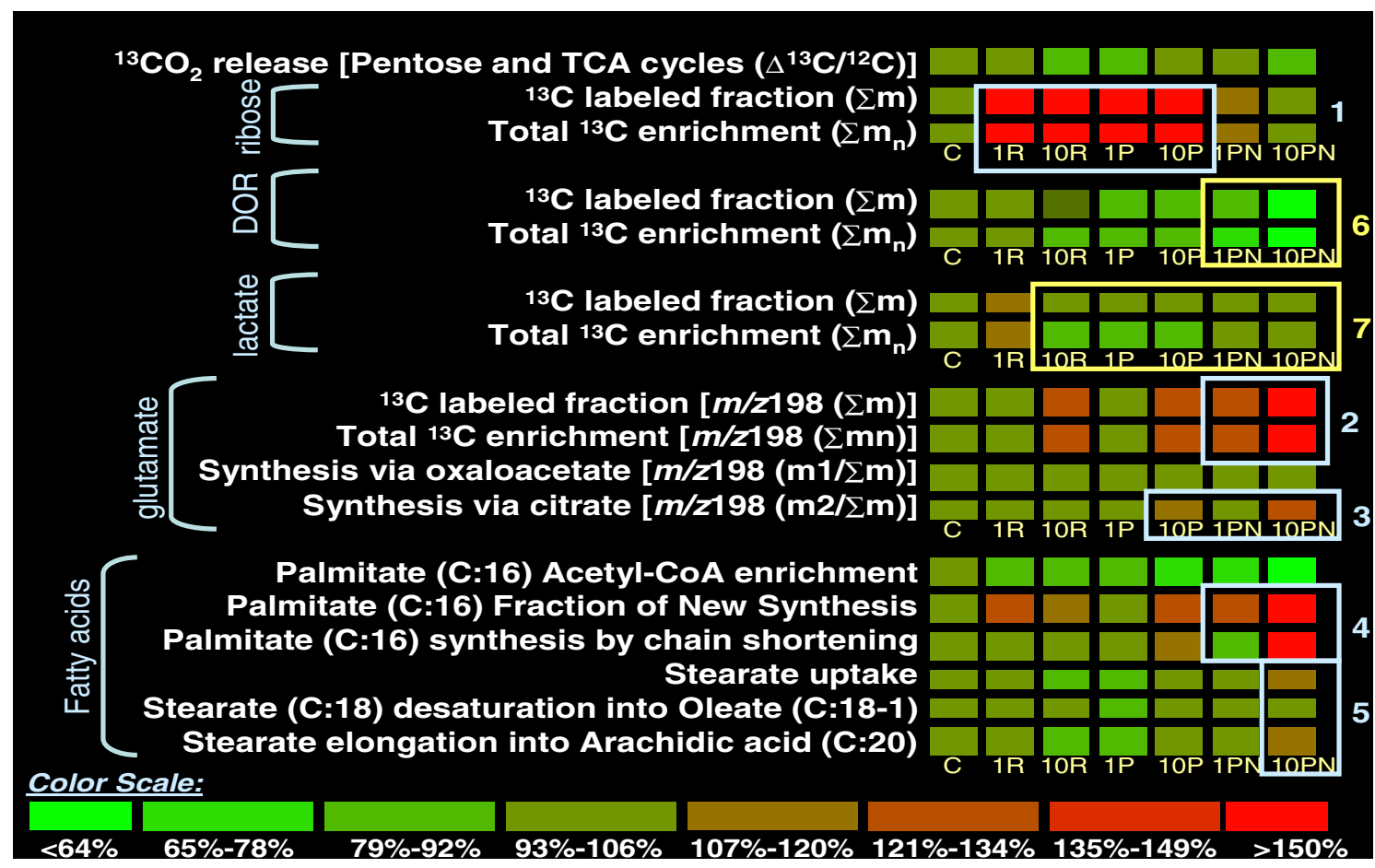

Figure 2. Stable isotope-based dynamic metabolic profile array (SIDMAParray) of rosiglitazone, pioglitazone and PNU-91325 in HepG2 cells using [U- ${ }^{13} \mathrm{C}_{18}$ ]stearic acid as the tracer. Treatments are indicated as follows: $\mathrm{C}=\mathrm{Control} ; 1 \mathrm{R}=1 \mu \mathrm{M}$ rosiglitazone; $10 \mathrm{R}=10 \mu \mathrm{M}$ rosiglitazone; $1 \mathrm{P}=1 \mu \mathrm{M}$ pioglitazone; $10 \mathrm{P}=10 \mu \mathrm{M}$ pioglitazone; $1 \mathrm{PN}=1 \mu \mathrm{M}$ PNU-91325; and $10 \mathrm{PN}=10 \mu \mathrm{M}$ PNU-91325. The color of each square corresponds to ${ }^{13} \mathrm{C}$ carbon flux from stearate via various synthetic pathways of ribose, deoxyribose, lactate, glutamate of the TCA cycle and fatty acids as percent of control-DMSO treated cultures. A color scheme indicating the intensity of changes in substrate flux is provided in the bottom of the figure. $(\mathrm{DOR}=$ deoxyribose; G6PDH = glucose 6-phosphate dehydrogenase; TPI = Triose-phosphate isomerase). 


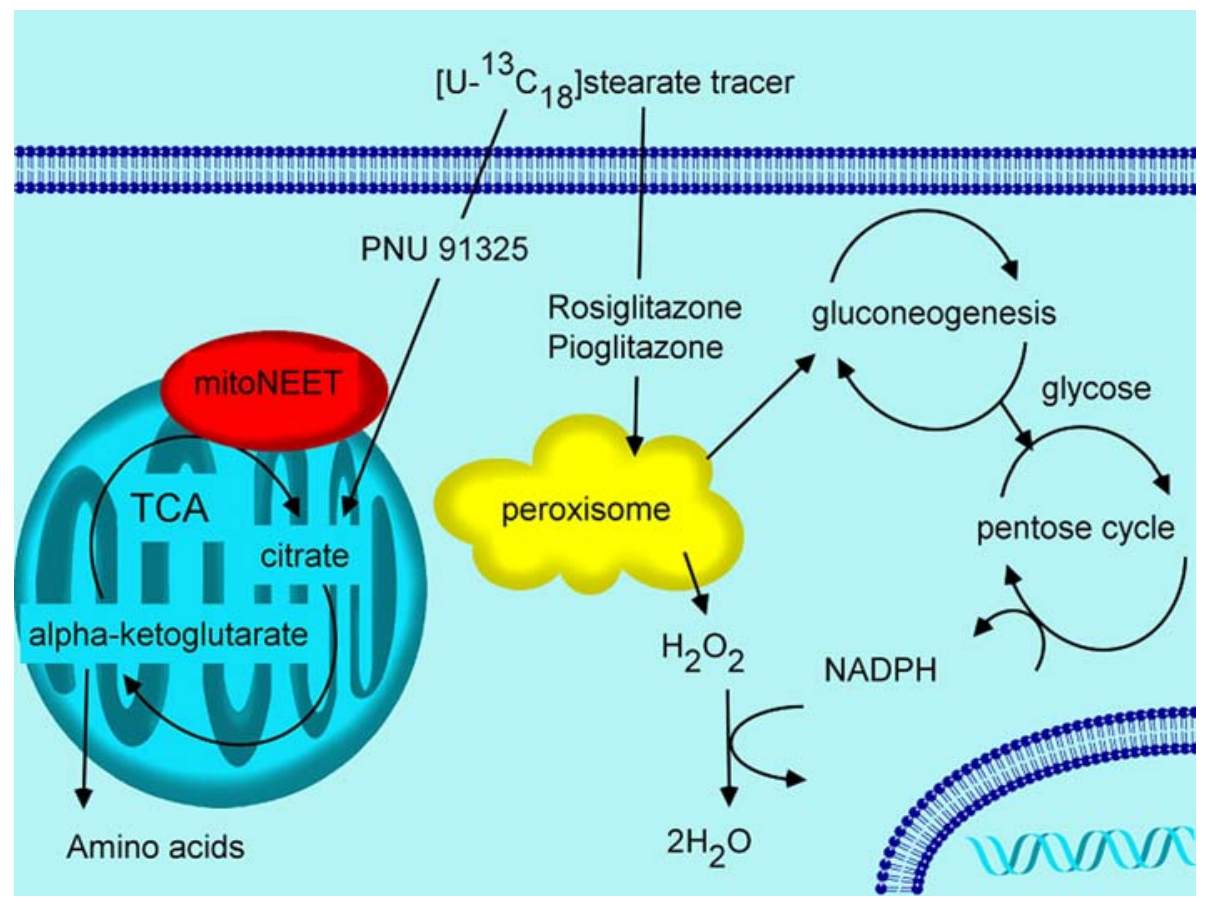

Figure 3. Substrate flow and metabolome action sites of rosiglitazone, pioglitazone and PNU-91325 in HepG2 cells. Rosiglitazone and pioglitazone concomitantly increase peroxisomal long chain fatty acid degradation while shifting acetyl- and acyl-CoA flux towards hexose and pentose synthesis. PNU-91325 forces long chain fatty acid oxidation to occur in the mitochondria and acetyl-CoA disposal via glutamate synthesis. The acetyl-CoA pool of the mitochondria generated by chain shortening of stearate in response to mitoNEET activation also becomes the source of chain elongation of fatty acids (not shown here).

various cells, a tracer study in vivo is warranted using glucose and stearate tracer enriched diets in a wild type and diabetic host in order to better understand the effect of a primarily mitochondrially acting TZD (via mitoNEET?) plays in the regulation of long chain fatty acid breakdown and metabolism as compared to the currently marketed TZDs that are more potent PPAR $\gamma$ activators.

Finally, it is evident that many reported effects of TZDs are clearly independent of PPARs. A most recent example is the demonstration that TZD inhibition of iNOS expression does not require PPAR involvement (Crosby et al., 2005). We suggest that many reported effects of TZD actions as being primarily mediated through PPAR should be interpreted with caution and that further investigation of the many secondary pharmacological effects associated with TZDs could contribute to the development of new therapeutic approaches to diabetes.

\section{References}

Boros, L.G., Cascante, M. and Lee, W.N. (2002). Metabolic profiling of cell growth and death in cancer: applications in drug discovery. Drug Discov. Today 7, 364-372.

Boros, L.G., Serkova, N.J., Cascante, M. and Lee, W.N. (2004). Use of metabolic pathway flux information in targeted cancer drug design. Drug Discov. Today Therap. Strategies 1, 435-443.

Colca, J.R., McDonald, W.G., Waldon, D.J., Leone, J.W., Lull, J.M., Bannow, C.A., Lund, E.T. and Mathews, W.R. (2004). Identification of a novel mitochondrial protein ("mitoNEET") cross-linked specifically by a thiazolidinedione photoprobe. Am. J. Physiol. Endocrinol. Metab. 286, E252-E260.

Colca, J.R. (2006). Metabolic inflammation and the insulin sensitizers. Biochem. Pharmacol. in press.

Crosby, M.B., Svenson, J.L., Zhang, J., Nicol, C.J., Gonzalez, F.J. and Gilkeson, G.S. (2005). Peroxisome proliferation-activated receptor $(\operatorname{PPAR} \gamma)$ is not necessary for synthetic $\operatorname{PPAR} \gamma$ agonist inhibitor of inducible nitric-oxide synthase and nitric oxide. J. Pharm. Exp. Therap. 312, 69-76.

Darmaun, D., Matthews, D.E., Desjeux, J.F. and Bier, D.M. (1988). Glutamine and glutamate nitrogen exchangeable pools in cultured fibroblasts: a stable isotope study. J. Cell Physiol. 134, 143-148.

Edvardsson, U., Bergstrom, M., Alexandersson, M., Bamberg, K., Ljung, B. and Dahllof, B. (1999). Rosiglitazone (BRL49653), a PPAR $\gamma$-selective agonist, causes peroxisome proliferator-like liver effects in obese mice. J. Lipid Res. 40, 1177-1184.

Guo, Y.T., Leng, X.S., Li, T., Zhao, J.M. and Lin, X.H. (2004). Peroxisome proliferator-activated receptor gamma ligands suppress liver carcinogenesis induced by diethylnitrosamine in rats. World J. Gastroenterol. 10, 3419-3423.

Haber, S. and Lapidot, A. (2001). Energy fuel utilization by fetal versus young rabbit brain: a ${ }^{13} \mathrm{C}$ MRS isotopomer analysis of $\left[\mathrm{U}-{ }^{13} \mathrm{C}_{6}\right.$ ]glucose metabolites. Brain Res. 896, 102-117.

Harrigan, G.G., Brackett, D.J. and Boros, L.G. (2005). Medicinal chemistry, metabolic profiling and drug target discovery: a role for metabolic profiling in reverse pharmacology and chemical genetics. Mini. Rev. Med. Chem. 5, 13-20.

Hellerstein, M.K. (1991). Relationship between precursor enrichment and ratio of excess M2/excess M1 isotopomer frequencies in a secreted polymer. J. Biol. Chem. 266, 10920-10924.

Katz, J., Wals, P. and Lee, W.N. (1993). Isotopomer studies of gluconeogenesis and the Krebs cycle with 13C-labeled lactate. J. Biol. Chem. 268, 25509-25521. 
Lee, W.N., Byerley, L.O., Bergner, E.A. and Edmond, J. (1991). Mass isotopomer analysis: theoretical and practical considerations. Biol. Mass Spectrom. 20, 451-458.

Lee, W.N., Bergner, E.A. and Guo, Z.K. (1992). Mass isotopomer pattern and precursor-product relationship. Biol. Mass Spectrom. 21, 114-122.

Lee, W.N., Edmond, J., Bassilian, S. and Morrow, J.W. (1996). Mass isotopomer study of glutamine oxidation and synthesis in primary culture of astrocytes. Dev. Neurosci. 18, 469-477.

Lee, W.N., Lim, S., Bassilian, S., Bergner, E.A. and Edmond, J. (1998a). Fatty acid cycling in human hepatoma cells and the effects of troglitazone. J. Biol. Chem. 273, 20929-20934.

Lee, W.N., Boros, L.G., Puigjaner, J., Bassilian, S., Lim, S. and Cascante, M. (1998b). Mass isotopomer study of the nonoxidative pathways of the pentose cycle with $\left[1,2-{ }^{13} \mathrm{C} 2\right]$ glucose. Am. J. Physiol. 274, E843-851.

Leimer, K.R., Rice, R.H. and Gehrke, C.W. (1977). Complete mass spectra of $N$-trifluoroacetyl- $n$-butyl esters of amino acids. J. Chromatogr. 141, 121-144.

Lowenstein, J.M., Brunengraber, H. and Wadke, M. (1975). Measurement of rates of lipogenesis with deuterated and tritiated water. Methods Enzymol. 35, 279-287.

Osawa, E., Nakajima, A., Wada, K., Ishimine, S., Fujisawa, N., Kawamori, T., Matsuhashi, N., Kadowaki, T., Ochiai, M.,
Sekihara, H. and Nakagama, H. (2003). Peroxisome proliferatoractivated receptor gamma ligands suppress colon carcinogenesis induced by azoxymethane in mice. Gastroenterology 124, 361-367.

Otto, C., Lehrke, M. and Goke, B. (2002). Novel insulin sensitizers: pharmacogenomic aspects. Pharmacogenomics 3, 99-116.

Sabate, L., Franco, R., Canela, E.I., Centelles, J.J. and Cascante, M. (1995). A model of the pentose phosphate pathway in rat liver cells. Mol. Cell. Biochem. 142, 9-17.

Tanis, S.P., Parker, T.T., Colca, J.R., Fisher, R.M. and Kletzein, R.F. (1996). Synthesis and biological activity of metabolites of the antidiabetic, antihyperglycemic agent pioglitazone. J. Med. Chem. 39, 5053-5063.

Vance, D.E. and Vance, K.E. (2002). Biochemistry of Lipids, Lipoproteins and Membranes. (fourth ed.). Elsevier Science Publishing Co., Inc., New York.

Wilkening, S., Stahl, F. and Bader, A. (2003). Comparison of primary human hepatocytes and hepatoma cell line Hepg2 with regard to their biotransformation properties. Drug Metab. Dispos. 31, $1035-1042$.

Wong, D.A., Bassilian, S., Lim, S. and Lee, W.N. (2004). Coordination of peroxisomal beta-oxidation and fatty acid elongation in HepG2 cells. J. Biol. Chem. 279, 41302-41309. 